\title{
Center-of-pressure total trajectory length is a complementary measure to maximum excursion to better differentiate multidirectional standing limits of stability between individuals with incomplete spinal cord injury and able-bodied individuals
}

Jean-François Lemay ${ }^{1,2}$, Dany H Gagnon ${ }^{1,2^{*}}$, Sylvie Nadeau ${ }^{1,2}$, Murielle Grangeon ${ }^{1,2}$, Cindy Gauthier ${ }^{1,2}$ and Cyril Duclos ${ }^{1,2}$

\begin{abstract}
Background: Sensorimotor impairments secondary to a spinal cord injury affect standing postural balance. While quasi-static postural balance impairments have been documented, little information is known about dynamic postural balance in this population. The aim of this study was to quantify and characterize dynamic postural balance while standing among individuals with a spinal cord injury using the comfortable multidirectional limits of stability test and to explore its association with the quasi-static standing postural balance test.

Methods: Sixteen individuals with an incomplete spinal cord injury and sixteen able-bodied individuals participated in this study. For the comfortable multidirectional limits of stability test, participants were instructed to lean as far as possible in 8 directions, separated by $45^{\circ}$ while standing with each foot on a forceplate and real-time COP visual feedback provided. Measures computed using the center of pressure (COP), such as the absolute maximal distance reached $\left(\mathrm{COP}_{\max }\right)$ and the total length travelled by the $\mathrm{COP}$ to reach the maximal distance $\left(C O \mathrm{P}_{\text {length }}\right)$, were used to characterize performance in each direction. Quasi-static standing postural balance with eyes open was evaluated using time-domain measures of the COP. The difference between the groups and the association between the dynamic and quasi-static test were analyzed.

Results: The COP length of individuals with $\mathrm{SCl}$ was significantly greater $(p \leq 0.001)$ than that of able-bodied individuals in all tested directions except in the anterior and posterior directions ( $p \leq 0.039)$, indicating an increased COP trajectory while progressing towards their maximal distance. The COP $\max$ in the anterior direction was significantly smaller for individuals with SCl. Little association was found between the comfortable multidirectional limits of stability test and the quasi-static postural balance test $(r \geq-0.658)$.

(Continued on next page)
\end{abstract}

\footnotetext{
* Correspondence: dany.gagnon.2@umontreal.ca

'School of Rehabilitation, Université de Montréal, Montreal, Canada

${ }^{2}$ Pathokinesiology Laboratory, Centre for Interdisciplinary Research in

Rehabilitation of Greater Montreal, Institut de réadaptation Gingras-Lindsay-

de-Montréal, 6300 avenue Darlington Montreal, QC H3S 2J4, Canada
} 


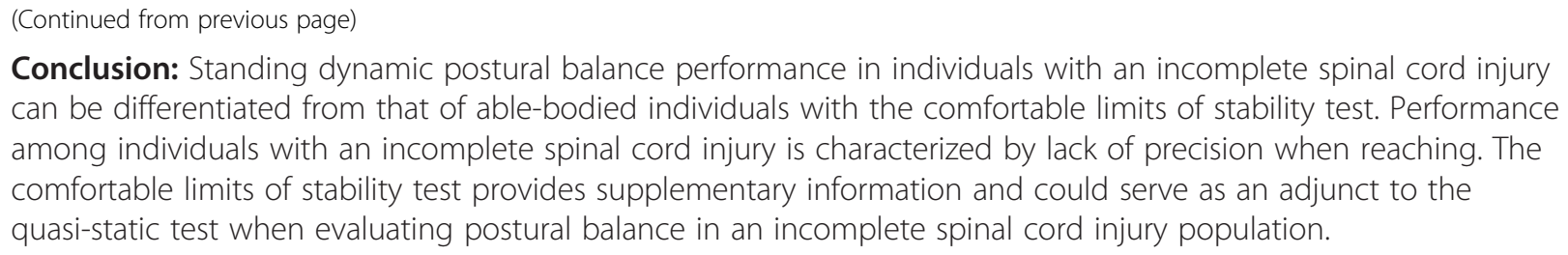

Keywords: Movement, Outcome assessment, Postural balance, Rehabilitation, Spinal cord injuries

\section{Background}

Following an incomplete spinal cord injury (SCI), most individuals will experience sensory and motor impairments at and below the level of the lesion. Due to the neural configuration of the spinal cord, an incomplete SCI will generally result in various degrees of lower extremity, trunk and upper extremity impairments depending on the level and the neural structures specifically affected by the lesion. These impairments often impact the ability to stand safely and execute functional activities in this position (e.g., multidirectional reaching). In fact, recent figures report a high incidence of falls (up to 75\%) in individuals with an incomplete SCI, often resulting in physical injuries and decreased social participation [1,2]. Individuals with SCI identified loss of balance as one of the major factors contributing to falls [2]. Unfortunately, only a few studies have investigated how people with SCI control postural balance during quiet standing $[3,4]$ and how it changes when the trunk, head and upper extremities are engaged in dynamic movements over a fixed base of support [4].

Postural balance could be defined as the ability to stabilize the body's center of mass (COM) over its base of support (BOS) [5]. To preserve a state of balance, the COM position is constantly regulated by the position of the center of pressure (COP), defined as the point location of the ground reaction force vector on the surface of a force platform on which a person is standing [6]. Given the stabilizing role of the COP, numerous valid and reliable parameters $[6,7]$ have been recommended to characterize performance during quasi-static stance (i.e., standing with no intended movement [8]. So far, evidence of quasi-static postural balance impairments in individuals with SCI has been demonstrated. For instance, these individuals are less stable when standing either with their eyes open or closed than able-bodied individuals [3]. Moreover, their use of visual information to maintain quasi-static stance is greater compared to healthy individuals [3]. However, a comprehensive assessment of postural balance should include quasi-static stance and dynamic activities since performance in both conditions does not usually correlate in healthy adults $[9,10]$; nevertheless, this issue remains controversial [11]. The signs of dynamic instability have been documented during walking in this population and have been mostly expressed by greater variability in the margin of stability, step length, step width and mediolateral and anteroposterior foot placement as compared to controls [12]. To quantify dynamic balance during functional activities, other comprehensive biomechanical models have also been proposed but have yet to be used in individuals with SCI [13-15].

Another method for assessing dynamic postural balance is by exploring the limits of stability, which can be defined as the maximum distance an individual is willing to move his/her centre of pressure (COP) in various directions without changing the configuration of the BOS and while remaining stable [16]. This usually involves the use of a forceplate that records COP displacement and a visual display that serves as feedback to maximize COP movement in specific directions. A multidirectional limits of stability test has recently been used to characterize dynamic sitting postural balance in individuals with SCI $[17,18]$. Individuals having no motor control over their abdominal and low back muscles had distinct ability on this test compared to those who had partial or full control, which tends to support its validity in a seated position [17]. However, standing imposes different biomechanical and dynamic constraints on stability compared to a sitting position, provided that, for example, the BOS is smaller and the COM higher. An analysis of the COP while standing in healthy young adults reveals that time-domain measures were larger and frequency-domain measures were smaller compared to sitting, an observation attributed to the body segment that is moving in each position [19]. The multidirectional limits of stability test thus requires further study before it can be recommended for evaluating postural balance while standing.

Therefore, we conducted a comparative study using a laboratory-based measure of dynamic postural balance, i.e., the comfortable multidirectional standing test, as well as a quasi-static postural stability test in both spinal cord injury and able-bodied individuals. The main purpose of this study was to quantify standing dynamic postural balance in individuals with SCI. The secondary purpose of this study was to measure the association of this test with quasi-static standing balance. We hypothesized that individuals with SCI would present a lower level of dynamic postural balance while standing as compared to able-bodied individuals. We also expected a 
low level of association with the quasi-static standing postural balance test.

\section{Methods}

Participants

Sixteen individuals with an incomplete traumatic SCI (American Spinal Cord Injury (ASIA) Impairment Scale = D) [20] and 16 able-bodied controls volunteered to participate in this study. Participants with SCI were recruited from both the inpatient and outpatient population of the SCI rehabilitation unit of the Institut de réadaptation Gingras-Lindsay-de-Montréal (IRGLM). All SCI participants could stand for 5 minutes without external support and walk independently for $10 \mathrm{~m}$ with or without a walking assistive device. Participants were excluded if they presented other concomitant neurological conditions in addition to the SCI or walking or balance difficulties prior to the SCI. None of the able-bodied participants reported having musculoskeletal or neurological impairments that would interfere with standing postural balance. Ethics approval was obtained from Research Ethics Committee of the Center for Interdisciplinary Research in Rehabilitation of Greater Montreal (CRIR-5780111). Written consent was obtained after participants had read and understood the information about the research (Table 1).

\section{Clinical assessment}

Demographic information pertaining to the date and type of accident, ASIA level and the presence of any relevant associated conditions were gathered from each subject's chart. A physical therapist with 10 years of experience in the field of SCI rehabilitation conducted the lower extremity motor score assessment (LEMS) according to ASIA standards [20]. Natural walking speed was tested over a distance of $15 \mathrm{~m}$. Participants were asked to walk at their usual walking speed without any walking assistive devices [21]. The middle $10 \mathrm{~m}$ section was timed using a stopwatch. The task was repeated three times and the resulting speeds were averaged.

\section{Laboratory assessment}

All evaluations were conducted at the Pathokinesiology Laboratory of the Centre for Interdisciplinary Research in Rehabilitation of Greater Montreal (CRIR) located at the IRGLM. During the laboratory assessment, participants stood on two side-by-side forceplates embedded in the floor with their feet in a standardized position (heels $10 \mathrm{~cm}$ apart; feet abducted $20^{\circ}$ ) and their arms resting alongside their trunk.

\section{Comfortable multidirectional limits of stability test}

Following a familiarization period, participants were asked to lean from the starting position as far as possible at a comfortable self-selected speed in eight specific directions, each separated by $45^{\circ}$ (anterior, right anterolateral, right, right posterolateral, posterior, left posterolateral, left, left anterolateral) and return to the initial position within a 15-second period. Participants were instructed to keep their arms alongside their trunk, to initiate the movement from the ankle instead of bending their trunk or their hips when leaning and to avoid raising their toes or heels [22]. These instructions were given to facilitate COP movement in the indicated direction within a standardized BOS and to limit compensatory movements. A flat screen placed $2 \mathrm{~m}$ in front of the participant displayed the real-time position of their COP as well as the boundaries within which they had to move. Each of the eight directions was tested twice for a total of 16 movements that were displayed in a random order determined by a computer. A research engineer coordinated the computerized data acquisition and storage at all times.

\section{Quasi-static postural stability test}

Participants were asked to stand still during two 45-second trials with their eyes open. Participants had to keep their feet in the same standardized position as described above [3].

\section{Data processing}

Reaction forces were recorded for each task at a sampling frequency of $600 \mathrm{~Hz}$. The resultant COP time

Table 1 Descriptive characteristics of the participants $(n=32)$, mean $(S D)$ and range

\begin{tabular}{|c|c|c|c|c|}
\hline & \multicolumn{2}{|c|}{$\mathrm{SCl}$ group $(n=16)$} & \multicolumn{2}{|c|}{ Able-bodies group $(n=16)$} \\
\hline & Mean $(S D)$ & Range & Mean $(S D)$ & Range \\
\hline Age (years)* & $50.3(17.4)$ & $20-67$ & $41.5(13.2)$ & $23-67$ \\
\hline Height $(\mathrm{cm})^{*}$ & $173.4(6.5)$ & $158-183$ & $174.5(6.4)$ & $165-189$ \\
\hline $\operatorname{Mass}(\mathrm{kg})^{*}$ & $79.6(15.8)$ & $53.6-105.5$ & $83.4(13.2)$ & $65.8-123.5$ \\
\hline Time post lesion (days) & $318.3(226.8)$ & $15-740$ & & \\
\hline $\operatorname{LEMS}(/ 50) n=14$ & $45.3(3.6)$ & $39-50$ & & \\
\hline Natural speed (m/s) & $1.02(0.27)$ & $0.53-1.39$ & & \\
\hline
\end{tabular}


series, computed from the tri-axial components of the combined reaction forces, was filtered with a fourth-order Butterworth zero-lag filter with a cut-off frequency of $5 \mathrm{~Hz}$ and then down-sampled $(300 \mathrm{~Hz})$ before analysis.

\section{Outcome measures}

Comfortable multidirectional limits of stability test

Three main outcome measures were taken:

- $\mathrm{COP}_{\max }$ : represents the linear distance in $\mathrm{mm}$ between the initial and maximal positions of the $\mathrm{COP}$ in a given direction. The $\mathrm{COP}_{\max }$ was calculated for each trial in the eight directions tested. Greater $\mathrm{COP}_{\max }$ indicates better ability to move the COP in a specific direction.

- $\mathrm{COP}_{\text {length }}$ : represents the total trajectory in $\mathrm{mm}$ followed by the COP from its initial position to its maximal position. The $\mathrm{COP}_{\text {length }}$ was also computed for each of the eight directions. Lower $\mathrm{COP}_{\text {length }}$ indicates a straighter movement when reaching in a specific direction, possibly indicating better precision of the COP movement.

- $\mathrm{COP}_{\text {area: }}$ represents the area in $\mathrm{mm}^{2}$ defined by an ellipse fitting the $\mathrm{COP}_{\max }$ in each of the 8 tested directions. Greater $\mathrm{COP}_{\text {area }}$ indicates better overall ability to move the COP in every direction.

The mean COP speed was also calculated for each trial by taking the total distance travelled between the COP starting and maximal $\left(\mathrm{COP}_{\max }\right)$ positions divided by the time taken to reach this distance. Each trial was then analyzed to be included in the final analysis. Data from trials where a loss of balance or a foot displacement occurred were also discarded. The angular error (representing the absolute difference in degrees between the targeted direction and the actual $\mathrm{COP}_{\max }$ direction) was calculated for each trial. Only trials with an angular error $\leq 25^{\circ}$ were included in the analysis to minimize the impact of imprecise COP directions. For each direction, the maximal value of the $\mathrm{COP}_{\max }$ was taken as well as the corresponding $\mathrm{COP}_{\text {length }}$.

\section{Quasi-static postural stability test}

The mean values of the root mean square distance (RMS; $\mathrm{mm}$ ), the mean COP velocity (MV; $\mathrm{mm} / \mathrm{s}$ ), and the COP sway area $\left(\mathrm{SA} ; \mathrm{mm}^{2} / \mathrm{s}\right)$ were computed for each trial from the COP time series [3,6,7]. Lower RMS, MV and SA values would indicate a better performance in terms of postural balance. The mean of the two trials was calculated for each parameter.

\section{Data analysis}

Descriptive statistics (mean, standard deviation, range) were calculated for the participants' characteristics and all laboratory outcome measures. Normality of the distribution was explored using the Shapiro-Wilk test. Among the comfortable multidirectional limits of stability test parameters, the $\mathrm{COP}_{\text {length }}$ in the right, right posterolateral, posterior and left directions of individuals with $\mathrm{SCI}$, as well as the $\mathrm{COP}_{\text {length }}$ in the anterior and right posterolateral directions of the able-bodied participants were not normally distributed $(\mathrm{p} \leq 0.032)$. The quasistatic postural stability test departed from normality in individuals with $\mathrm{SCI}$ for the MV $(\mathrm{p} \leq 0.037)$. Nonparametric statistics were thus used when analyzing these data. Otherwise, all other data were normally distributed among the two groups and analyzed with parametric tests.

Independent $\mathrm{t}$-tests were used to reveal differences in the demographic data of both groups of participants. For the comfortable multidirectional limits of stability test parameters, a two-way repeated measure analysis of variance (ANOVA) with one between factor (individuals with SCI and able-bodied individuals) and one within factor (eight tested directions) was conducted to detect the presence of significant differences. Since there was a significant interaction between the $\mathrm{COP}_{\max }$ and the groups, independent t-tests or Mann-Whitney $U$ tests were performed to reveal between-group differences for each direction, using a Bonferonni correction on the alpha value $(p=0.05 / 8$ tested directions). Effect size were calculated for both the $\mathrm{COP}_{\max }$ and the $\mathrm{COP}_{\text {length }}$ and were considered as being small (0.2 to 0.5$)$, moderate (0.5-0.8) or large $(>0.8)$ according to Cohen's criteria [23]. Difference between the groups on the quasi-static test was quantified using independent t-test or Mann-Whitney $U$ test according to the normality of the data. Further analysis on the $\mathrm{COP}_{\max }$ where individuals with SCI were better than able-bodied controls was done by assessing the level of association with the $\mathrm{COP}_{\text {length }}$ in the same direction using Pearson's product moment correlation coefficient. The betweengroup difference of the $\mathrm{COP}_{\text {area }}$ and the mean COP speed was assessed with an independent $\mathrm{t}$-test.

The level of association between the comfortable multidirectional limits of stability test and the quasistatic postural stability test for individuals with SCI was explored using Pearson's product moment correlation coefficients. Correlation coefficients confirmed good to excellent association when they were greater than 0.75 [24]. A statistical significance threshold was set at 0.05 for all tests unless otherwise specified. All statistical analyses were conducted using SPSS ${ }^{\circ}$ software (version 20.0) (Chicago, IL).

\section{Results}

Between-group and within-group differences

For the comfortable multidirectional limits of stability test, a significant difference was found only in the anterior 
direction for the $\mathrm{COP}_{\max }$ between the two groups of participants at a statistical significance level of 0.00625 . However, individuals with SCI tended to have a greater $\mathrm{COP}_{\max }$ when reaching laterally and posteriorly (12.1 to 17.5\%). Able-bodied individuals had greater $\mathrm{COP}_{\max }$ when reaching in anterior directions (9.3 to $29.5 \%$ ).

The $\mathrm{COP}_{\text {length }}$ for individuals with SCI was 22.2-35.8\% greater than those of able-bodied individuals. This reached the significance level in all directions $(\mathrm{p} \leq 0.001)$, except in the anterior and posterior direction $(\mathrm{p} \leq 0.039)$. Both the $\mathrm{COP}_{\text {max }}$ and the $\mathrm{COP}_{\text {length }}$ tended to be smaller in the posterior directions as compared to anterior and lateral directions (Figures 1 and 2). Effect sizes were considered moderate to large.

There was a significant level of association between the $\mathrm{COP}_{\text {max }}$ and the $\mathrm{COP}_{\text {length }}$ in the posterior, left posterolateral and left directions for individuals with SCI $(0.527 \leq \mathrm{r} \leq 0.630, \mathrm{p} \leq 0.036)$.

The $\mathrm{COP}_{\text {area }}$ was not significantly different between SCI and able-bodied participants $(\mathrm{p}=0.560)$ (Table 2). No difference was seen on the mean COP speed at the significance level of 0.00625 . All COP parameters of the quasi-static postural stability test revealed reduced postural balance in individuals with SCI as compared to able-bodied participants $(\mathrm{p} \leq 0.0002)$.

\section{Correlation between the comfortable multidirectional limits of stability test and the quasi-static postural stability test for individuals with $\mathrm{SCl}$}

For individuals with SCI, there was a limited number of significant associations (4 out of 48 possible associations) between the parameters of the comfortable multidirectional limits of stability test and the quasi-static postural stability test (Table 3). A moderate level of association was present between the $\mathrm{COP}_{\max }$ in a left anterolateral direction and the $\mathrm{MV}$ and SA parameter of the quasi-static postural stability test $(-0.658 \leq \mathrm{r} \leq-0.559 ; \mathrm{p} \leq 0.014)$, as well as between the $\mathrm{COP}_{\text {length }}$ in the right anterolateral direction and the RMS and MV parameters $(0.501 \leq \mathrm{r} \leq$ 0.602; $\mathrm{p} \leq 0.048$ ) (Figure 3).

\section{Discussion}

In this study, we have found that a task testing the limits of stability in SCI individuals who are community ambulators, is mainly characterized by an increase in the distance travelled by the COP when going to its maximal position rather than by a limitation in the absolute distance reached by the COP in the intended direction. Therefore, using $\mathrm{COP}_{\text {length }}$ may better characterize performance of individuals with SCI. When the $\mathrm{COP}_{\max }$ is greater in individuals

A)
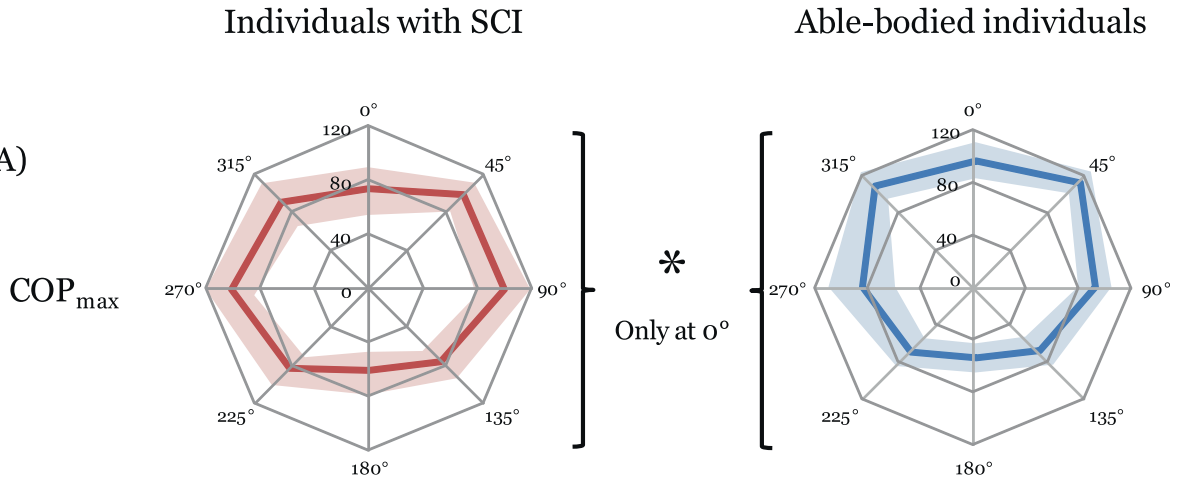

B)

$\mathrm{COP}_{\max }$

Able-bodied individuals
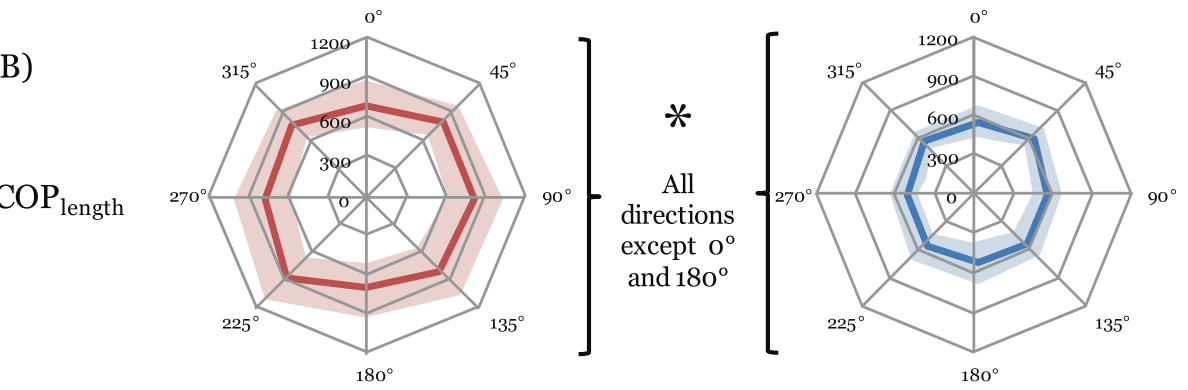

Figure 1 Comparison of performance for two parameters of the comfortable multidirectional limits of stability test between individuals with a spinal cord injury and able-bodied individuals. AL: anterolateral; PL: posterolateral. A) The COP max represents the maximal distance reached by the center of pressure in every direction. Although differences in performance between the groups can be seen in most directions, none reached the adjusted level of statistical differences $(0.00625$ (0.05/8 directions)). B) The COPlength represents the length of the COP trajectory from the starting position to the maximal position in a given direction. Individuals with $\mathrm{SCl}$ had significantly longer $\mathrm{COP}_{\text {length }}$ in all but one direction $\left(180^{\circ}\right)$ as compared to able-bodied individuals. 
Individual with SCI

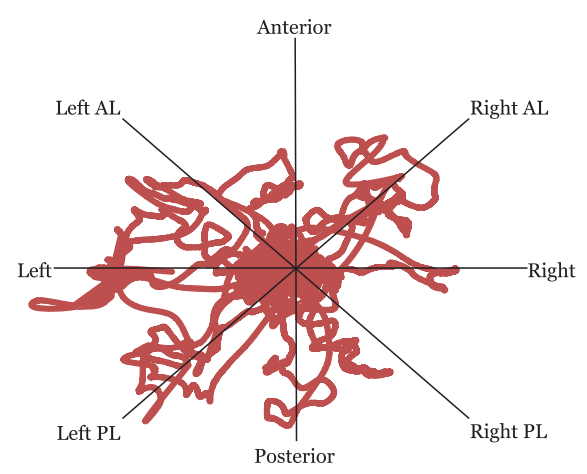

Able-Bodied Individual

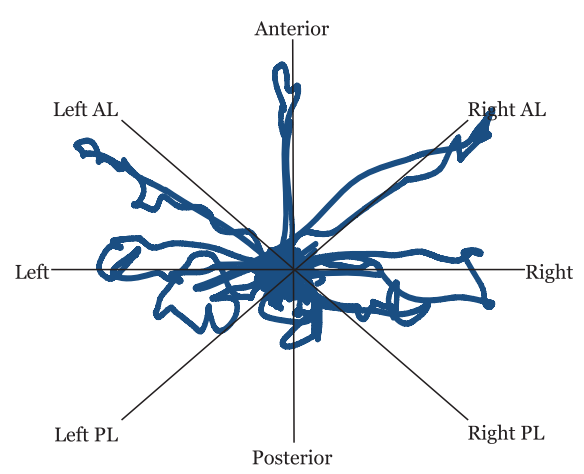

$100 \mathrm{~mm}$

Figure 2 An example of a COP displacement on the comfortable multidirectional limits of stability test performed by two individuals, one with and the other without SCI. AL: anterolateral; PL: posterolateral. The individual with SCI displays less precision when reaching in all directions as compared to the able-bodied individuals, for whom most directions are easier to differentiate. Overall, reaching movements in the posterior direction tended to be smaller and less precise than in the anterior and lateral directions in both groups.

Table 2 Descriptive characteristics of the participants on the dynamic and quasi-static tests

\begin{tabular}{|c|c|c|c|c|c|c|}
\hline \multirow[t]{2}{*}{ Test } & \multirow[t]{2}{*}{ Parameter } & \multirow[t]{2}{*}{ Specification } & \multirow{2}{*}{$\begin{array}{c}\mathrm{SCl} \text { group }(n=16) \\
\text { Mean }(S D)\end{array}$} & \multirow{2}{*}{$\begin{array}{c}\text { Able-bodied group }(n=16) \\
\text { Mean }(S D)\end{array}$} & \multicolumn{2}{|c|}{ Effect-size } \\
\hline & & & & & (d) & $(95 \% \mathrm{Cl})$ \\
\hline \multirow{17}{*}{$\begin{array}{l}\text { Comfortable multidirectional } \\
\text { limits of stability test }\end{array}$} & \multirow[t]{8}{*}{$\mathrm{COP}_{\max }(\mathrm{mm})$} & Anterior*十 & 75.5 (15.6) & $94.2(13.7)$ & 1.27 & $(0.48,2.00)$ \\
\hline & & Right AL & $100.6(12.0)$ & $110.0(11.7)$ & 0.79 & $(0.05,1.49)$ \\
\hline & & Right & $101.8(19.8)$ & $89.0(12.5)$ & 0.77 & $(0.04,1.47)$ \\
\hline & & Right PL & 76.5 (17.6) & $67.2(14.8)$ & 0.57 & $(-0.15,1.26)$ \\
\hline & & Posterior & $60.6(18.2)$ & $53.0(10.9)$ & 0.51 & $(-0.21,1.20)$ \\
\hline & & Left PL & 83.5 (17.9) & $68.9(14.7)$ & 0.89 & $(0.14,1.59)$ \\
\hline & & Left & $102.6(18.4)$ & $90.0(9.9)$ & 0.85 & $(0.11,1.55)$ \\
\hline & & Left AL & $92.4(19.3)$ & $106.7(14.2)$ & 0.84 & $(0.10,1.54)$ \\
\hline & \multirow[t]{8}{*}{$\mathrm{COP}_{\text {length }}(\mathrm{mm})$} & Anterior & $695.0(191.5)$ & $540.9(121.7)$ & 0.96 & $(0.21,1.67)$ \\
\hline & & Right $A L^{*}$ & $811.6(171.5)$ & 593.0 (99.6) & 1.56 & $(0.73,2.31)$ \\
\hline & & Right* $^{*}$ & $804.9(225.4)$ & $516.6(107.6)$ & 1.63 & $(0.80,2.39)$ \\
\hline & & Right $\mathrm{PL}^{*}+$ & $765.6(242.5)$ & $516.3(133.3)$ & 1.27 & $(0.49,2.00)$ \\
\hline & & Posterior & $659.2(220.9)$ & $502.9(173.2)$ & 0.79 & $(0.05,1.49)$ \\
\hline & & Left PL* & $831.7(221.7)$ & $537.4(161.4)$ & 1.52 & $(0.70,2.26)$ \\
\hline & & Left* & 749.6 (228.7) & $529.9(123.6)$ & 1.20 & $(0.42,1.91)$ \\
\hline & & Left AL* & 779.5 (156.8) & $569.4(87.3)$ & 1.66 & $(0.82,2.41)$ \\
\hline & $\mathrm{COP}_{\text {area }}\left(\mathrm{mm}^{2}\right)$ & & 20181.8 (4527.8) & $19332.4(3557.1)$ & 0.21 & $(-0.49,0.90)$ \\
\hline \multirow[t]{3}{*}{ Quasi-static test } & RMS (mm) & $\mathrm{EO}^{*}$ & $8.71(2.67)$ & $5.17(1.63)$ & 1.60 & $(0.77,2.35)$ \\
\hline & $\mathrm{MV}(\mathrm{mm} / \mathrm{s})$ & $\mathrm{EO}^{*}+$ & $16.00(6.20)$ & $7.52(2.00)$ & 1.84 & $(0.97,2.62)$ \\
\hline & $\mathrm{SA}\left(\mathrm{mm}^{2} / \mathrm{s}\right)$ & $\mathrm{EO}^{*} \dagger$ & $40.41(29.00)$ & $8.98(4.75)$ & 1.51 & $(0.69,2.26)$ \\
\hline
\end{tabular}

*Significant between-group difference (Independent t-test or Mann-Whitney $U$ test $(t) ; p<0.05)$. COP max: maximal distance reached by the COP in the indicated

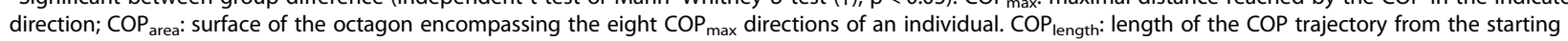
position to the maximal position of the COP; AL: anterolateral; PL: posterolateral; RMS: root mean square distance of the COP; MV: mean velocity of the COP; SA: Sway-area of the COP; EO: eyes open; $d$ : Cohen's $d$. 
Table 3 Correlation matrix between the dynamic and the quasi-static test for individuals with $\mathrm{SCl}$

\begin{tabular}{|c|c|c|c|c|c|c|c|c|}
\hline \multirow[t]{3}{*}{ Quasi-static test } & \multicolumn{8}{|c|}{ Comfortable multidirectional limits of stability test } \\
\hline & \multicolumn{8}{|c|}{$\mathrm{COP}_{\max }$} \\
\hline & Anterior & Right AL & Right & Right PL & Posterior & Left PL & Left & Left AL \\
\hline RMSt & -0.194 & 0.217 & -0.006 & 0.040 & 0.468 & 0.475 & 0.325 & -0.275 \\
\hline MV+ & -0.352 & 0.277 & -0.232 & -0.393 & -0.080 & 0.361 & 0.064 & $-0.658^{* *}$ \\
\hline \multirow[t]{3}{*}{ SAt } & -0.387 & 0.142 & -0.204 & -0.239 & 0.177 & 0.355 & 0.167 & $-0.559^{*}$ \\
\hline & \multicolumn{8}{|c|}{$\mathrm{COP}_{\text {length }}$} \\
\hline & Anterior & Right AL & Right & Right PL & Posterior & Left PL & Left & Left AL \\
\hline RMSt & -0.067 & $0.602^{*}$ & 0.365 & 0.004 & 0.441 & 0.138 & 0.241 & 0.366 \\
\hline MV† & 0.050 & $0.501^{*}$ & 0.173 & -0.133 & 0.290 & 0.190 & 0.091 & 0.081 \\
\hline SAt & 0.011 & 0.482 & 0.120 & -0.047 & 0.292 & 0.149 & 0.135 & 0.143 \\
\hline
\end{tabular}

†Pearson's product moment correlation coefficient. *: $p \leq 0.05,{ }^{* *}: p \leq 0.01$.

RMS: root mean square distance of the COP; MV: mean velocity of the COP; SA: sway area of the COP; COP max: maximal distance reached by the centre of pressure in the indicated direction. $\mathrm{COP}_{\text {length }}$ length of the COP trajectory from the starting position to the maximal position of the COP; AL: anterolateral; PL: posterolateral.

with SCI as compared to able-bodied individuals in some direction, it is associated with the $\mathrm{COP}_{\text {length }}$ which further support the use of this measure in a SCI population. To our knowledge, this is the first study to report such a finding in this population. In accordance with our second hypothesis, we also found little association between the quasi-static and the dynamic balance parameters.
Performance of individuals with $\mathrm{SCl}$ versus able-bodied individuals on the comfortable limits of stability test $\mathrm{COP}_{\text {length }}$ is calculated from the total excursion of the COP on its way toward its maximal position in an indicated direction. $\mathrm{COP}_{\text {length }}$ of individuals with $\mathrm{SCI}$ was greater than that of able-bodied individuals in all directions. This increased COP excursion could be interpreted as a decrease in movement precision: whereas
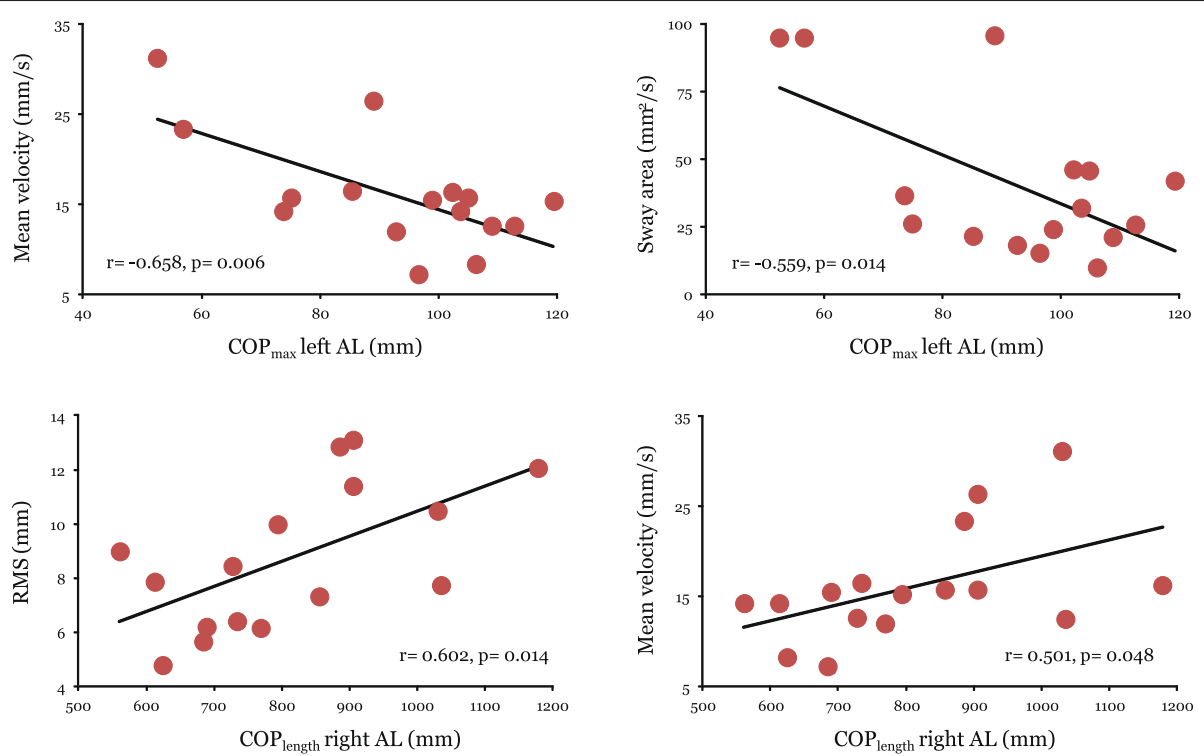

Figure 3 Scatter plots representing statistically significant associations between the comfortable multidirectional limits of stability test and the quasi-static postural steadiness test for individuals with $\mathrm{SCl}$. AL: anterolateral, RMS: Root mean square distance. Although the four relationships displayed are significant, they have to be interpreted with caution since all the other relationships between parameters of the quasi-static postural steadiness test and the comfortable multidirectional limits of stability test were not significant for individuals with SCI. Moreover, there seems to be a slight ceiling effect in terms of the sway area parameter in the quasi-static postural steadiness test seems on the second scatter plot. 
COP movement of able-bodied individuals tended to move in a straighter manner, individuals with SCI displayed more extraneous movements on their way to their maximal position, increasing the $\mathrm{COP}_{\text {length }}$ Similar results have been found in other populations presenting neurological deficits such as Parkinson's disease. Ondo et al. found that individuals with Parkinson's disease had a significantly increased path sway on the Smart Balance Master limits of stability test as compared to able-bodied individuals, a test comparable to the one performed in our study [25].

Many factors may be responsible for this decrease in movement precision during the comfortable multidirectional limits of stability test in individuals with SCI. Since a SCI generally alters the integrity of the various sensory tracts travelling within the spinal cord, the somatosensory contribution to postural balance may therefore be decreased, which in turn, could explain the less precise movement of the COP. Clinical evaluation of sensory function revealed residual sensory deficits in most of our participants with SCI. In fact, a varying degree of foot anesthesia induced from various techniques causes a proportional increase in COP motion while standing [26,27]. Moreover, an increase in visual contribution to postural balance in people with SCI as compared to able-bodied controls does exist [3]. This may represent a compensatory mechanism for a decreased sensory perception in the lower extremity. Future studies could identify to what extent sensory deficits following SCI is a major contributor to observed standing postural balance deficits.

Lower-extremity muscle function is another factor influencing control of standing postural balance in various populations [28-31]. Although our participants with SCI had sufficient lower extremity strength to assume a standing position, a certain deficit in strength was present in the ankle dorsiflexors and plantarflexors, as indicated by the LEMS evaluation. It is known that the location of lower-extremity muscle strength influences balance capability differently. For example, Horlings et al. demonstrated that distal muscle weakness more significantly influences postural stability than proximal muscle weakness [28]. Future studies using a dynamometric evaluation of lower extremities could help identify those muscle groups associated with increased $\mathrm{COP}_{\text {length }}$ in each direction.

Contrary to our initial hypothesis, individuals with SCI had similar $\mathrm{COP}_{\max }$ compared to able-bodied controls as indicated by the lack of significant differences in all but one direction and on the overall measure given by the $\mathrm{COP}_{\text {area }}$. Interestingly, these findings correspond to those of Gauthier et al. who showed that SCI individuals who had partial or full control over their abdominal and lower trunk muscles could bring their COP to a similar distance from their base of support to that of ablebodied individuals when performing a similar postural balance test while sitting [17]. This may seem surprising considering that standing is inherently less stable than sitting $[19,32]$ and could thus lead to greater differences in performance between impaired and normally functioning individuals. However in our study, individuals with SCI had adequate motor recovery in their trunk muscles to be able to assume a standing position and lean in various directions. This may in part explain the lack of difference seen in $\mathrm{COP}_{\max }$. Yet the significant groups vs. directions interaction indicates that individuals with SCI and able-bodied participants differed on how they performed in various directions. More specifically, individuals with SCI had greater $\mathrm{COP}_{\max }$ in lateral directions while able-bodied individuals had greater $\mathrm{COP}_{\text {max }}$ in anterior and antero-lateral directions. Complementary studies including a larger sample of participants could help to confirm whether or not a difference in $\mathrm{COP}_{\max }$ between the groups exists. Greater $\mathrm{COP}_{\text {max }}$ in some directions cannot be attributed to different foot placements since foot placements were standardized using a template. This is also supported by the absence of significant difference between the groups on the $\mathrm{COP}_{\max }$ in a posterior, right and left directions.

Ankle plantarflexor muscle groups are known to influence anteroposterior COP excursions [31] and are especially activated when controlling anterior body displacement with respect to the base of support [33]. Since most of our individuals with SCI had residual distal lower-extremity weakness, we could hypothesize that the lower $\mathrm{COP}_{\max }$ in the anterior and anterolateral directions may be explained by this lack of strength. Individuals with SCI could therefore limit anterior COP displacement in order to take into account their decreased ability to control the COP using their ankle plantarflexors when reaching the limits of stability in this direction.

On the other hand, individuals with SCI had greater $\mathrm{COP}_{\text {max }}$ in the lateral directions than able-bodied individuals. Body displacements in lateral directions are under the control of hip abductor muscle groups [33], which did not achieve full recovery in our group of individuals with SCI. A possible explanation for this better performance is that those individuals with SCI who outperformed ablebodied individuals in these directions did it at the expense of precision, as indicated by the $\mathrm{COP}_{\text {length }}$. This hypothesis is partly supported by the significant positive correlation found between the $\mathrm{COP}_{\max }$ and the $\mathrm{COP}_{\text {length }}$ in the posterior, left and left posterolateral directions where individuals with SCI were found to outperform able-bodied individuals. This indicates that, in these directions, individuals with SCI who could displace their COP further were generally less precise than those presenting more 
limited COP displacements. Since the comfortable multidirectional limits of stability test challenges the postural control system and attentional resources, some individuals may have favored moving less precisely in the displayed direction in order to achieve a maximal performance on the $\mathrm{COP}_{\max }$. Therefore, repeating this test while imposing no constraint on the movement precision may have yielded different $\mathrm{COP}_{\max }$ results.

\section{Association between quasi-static and dynamic postural balance tests}

This study also yields little association between dynamic and quasi-static postural balance for individuals with SCI. As mentioned earlier, only four of the 48 possible combinations were found to be statistically significant. This limits the inferences that can be drawn from these associations. These results support a previous study reporting a lack of significant correlation between the static eyes open test and the limits of stability test of the Smart Balance Master, the later sharing some similarities with the comfortable limits of stability test [4]. This is also in line with most studies exploring the association between measures of static and dynamic balance among individuals with stroke or able-bodied individuals [9,10,34]. In spite of these remarks, parameters of the COP during quasi-static stance correlated with the comfortable multidirectional limits of stability test in only two directions (left and right anterolateral directions). Further research including more participants with a wider range of balance deficits would be necessary to determine the significance of this specific result.

\section{Study limitations}

Our study suffers from a few limitations needing consideration when interpreting these results. Firstly, all our participants with SCI were community ambulators. Many participants with SCI had near normal walking ability, as indicated by the mean natural speed of $1.02 \mathrm{~m} / \mathrm{s}$, which is close to the $1.06 \mathrm{~m} / \mathrm{s}$ value required to be considered as a safe community ambulator [35]. It is thus possible that our group of SCI participants was not representative of an actual population of individuals with traumatic incomplete SCI. These results are therefore not applicable to those individuals who are starting to assume a standing position. This could have limited the possibility to find differences between our two groups. However, although statistical power of the study was in part limited by the small number of participants in each group, effect sizes were at least moderate for the $\mathrm{COP}_{\text {length }}$ in the comfortable multidirectional limits of stability test. Therefore, this statistic supports the fact that our sample size was sufficient to find differences between the groups.
As is often done in other studies, COP-based measures were not normalized using the dimension of the base of support or foot length $[17,18]$. However, our two groups did not differ in height. Since foot position was standardized and monitored during the study, we therefore presume that this normalization would not have changed our main conclusions. Although participants were told to initiate the movement from the ankle instead of bending their trunk or their hips when leaning, some degree of trunk and hip compensation of varying degrees was present among participants and directions. We thus suspect that the actual performance may be partially related to the strategies used. Adding a kinematic analysis to our protocol may have helped to identify biomechanical markers associated with the difference in performance seen among both groups.

It is possible that the performance of individuals with SCI is potentiated because of the visual feedback provided during this test. In fact, Sayenko et al. have shown that visual feedback can improve standing balance performance in individuals with incomplete SCI [36]. A test performed without visual feedback could have been more reflective of balance capabilities of each participant and could have generated more differences between the groups. Normal dynamic postural balance activities such as walking occur without on-screen visual feedback on actual performance. Therefore, how the results from this study can be generalized to other dynamic balance activities remains to be explored.

Although 15 seconds were given to maximally displace the COP in the indicated direction and come back to the initial position, no actual control on the speed of movement was given. However no difference was seen between the groups in the mean COP speed. Thus this factor could not explain the differences in performance (i.e., $\mathrm{COP}_{\text {length }}$ ) seen between the groups. We did not analyze the return from the maximal position to the initial position, which may have provided further insight into dynamic balance performance in individuals with SCI in the comfortable limits of stability test. Reliability and minimal detectable change of the $\mathrm{COP}_{\max }$ and the $\mathrm{COP}_{\text {length }}$ were not assessed. Since the standard error of measurement is unknown for these parameters, this limits the inferences that can be drawn from differences in performance seen between the groups of participants. Lastly, we did not apply a correction for multiple comparisons (e.g., Bonferonni's) to the correlational analysis. For this reason, the correlation that were significant must be interpreted with caution since a possibility of a type I error exists.

\section{Conclusion}

The comfortable multidirectional limits of stability test can characterize dynamic postural balance in individuals 
with SCI. More precisely, a measure of movement precision (i.e., $\mathrm{COP}_{\max }$ ) could differentiate the performance of SCI individuals from that of able-bodied controls. Although balance during quasi-static standing is impaired in individuals with SCI, no definitive association was found between this evaluation and the dynamic test under investigation. A comprehensive evaluation of postural balance should therefore include items assessing both its static and dynamic components.

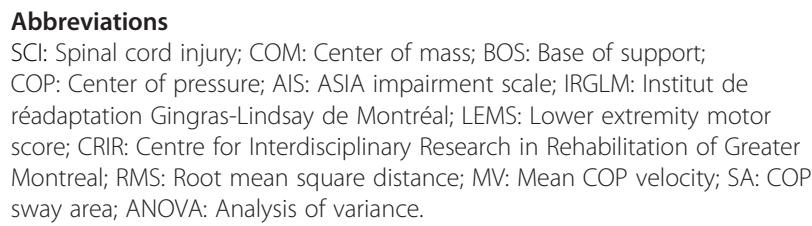

\section{Competing interests}

The authors declare that they have no competing interests.

\section{Authors' contributions}

JFL: Conception and design, acquisition, analysis and interpretation of data, drafted the article. DG: Conception and design, analysis and interpretation of data, drafted the article. SN: Conception and design, analysis and interpretation of data, drafted the article. MG: Conception and design, acquisition of data. CG: Conception and design, acquisition of data CD: Conception and design, acquisition of data. All authors read and approved the final manuscript.

\section{Authors' information}

School of Rehabilitation (www.readap.umontreal.ca), Université de Montréal, Montreal, Canada.

Pathokinesiology Laboratory (www.pathokin.ca), Centre for Interdisciplinary Research in Rehabilitation of Greater Montreal - Institut de réadaptation Gingras-Lindsay de Montréal, Montreal, Canada.

\section{Acknowledgements}

The authors acknowledge Philippe Gourdou, Youssef El Khamlichi, Carole Miéville, and Audrey Roy, for their assistance during data collection and analysis.

Jean-François Lemay is supported by a doctoral scholarship from the Fonds de la recherche du Québec-Santé (FRQS). Dany Gagnon holds a Junior 1 Career Award from the FRQS. Dany Gagnon, Cyril Duclos and Sylvie Nadeau are members of the Multidisciplinary SensoriMotor Rehabilitation Research Team (www.errsm.ca) supported by the CIHR and of the Quebec-Ontario Spinal Cord Injury Mobility Research Group. The equipment and material required to complete this project at the Pathokinesiology Laboratory was financed in part by the Canada Foundation for Innovation. This project was funded in part by a research grant from the Craig H. Neilson Foundation.

Received: 27 May 2013 Accepted: 3 January 2014

Published: 17 January 2014

\section{References}

1. Brotherton SS, Krause JS, Nietert PJ, Brotherton SS, Krause JS, Nietert PJ: A pilot study of factors associated with falls in individuals with incomplete spinal cord injury. J Spinal Cord Med 2007, 30:243-250.

2. Brotherton SS, Krause JS, Nietert PJ: Falls in individuals with incomplete spinal cord injury. Spinal Cord 2007, 45:37-40.

3. Lemay JF, Gagnon D, Duclos C, Grangeon M, Gauthier C, Nadeau S: Influence of visual inputs on quasi-static standing postural steadiness in individuals with spinal cord injury. Gait Posture 2013, 38:357-360.

4. Lemay JF, Nadeau S: Potential of the smart balance master system to assess the standing balance in people with AIS D spinal cord injury. J Rehabil Med 2013, 45:55-60.

5. Horak FB: Postural control. In Encyclopedia of Neuroscience. Edited by Binder MD, Hirokawa N, Windhorst U. Berlin Heidelberg: Springer; 2009:3212-3219.
6. Prieto TE, Myklebust JB, Hoffmann RG, Lovett EG, Myklebust BM: Measures of postural steadiness: differences between healthy young and elderly adults. IEEE Trans Biomed Eng 1996, 43:956-966.

7. Rocchi L, Chiari L, Cappello A: Feature selection of stabilometric parameters based on principal component analysis. Med Biol Eng Comput 2004, 42:71-79.

8. Hsiao-Wecksler ET, Katdare K, Matson J, Liu W, Lipsitz LA, Collins JJ: Predicting the dynamic postural control response from quiet-stance behavior in elderly adults. J Biomech 2003, 36:1327-1333.

9. Karimi MT, Solomonidis S: The relationship between parameters of static and dynamic stability tests. J Res Med Sci 2011, 16:530-535.

10. Kang HG, Dingwell JB: A direct comparison of local dynamic stability during unperturbed standing and walking. Exp Brain Res 2006, 172:35-48.

11. Abe MO, Masani K, Nozaki D, Akai M, Nakazawa K: Temporal correlations in center of body mass fluctuations during standing and walking. Hum Mov Sci 2010, 29:556-566.

12. Day KV, Kautz SA, Wu SS, Suter SP, Behrman AL: Foot placement variability as a walking balance mechanism post-spinal cord injury. Clin Biomech 2011, 13:13.

13. Duclos C, Desjardins P, Nadeau S, Delisle A, Gravel D, Brouwer B, Corriveau $H$ : Destabilizing and stabilizing forces to assess equilibrium during everyday activities. J Biomech 2009, 42:379-382.

14. Hof AL, Gazendam MG, Sinke WE: The condition for dynamic stability. J Biomech 2005, 38:1-8.

15. Pai YC, Patton J: Center of mass velocity-position predictions for balance control. J Biomech 1997, 30:347-354.

16. Holbein-Jenny MA, McDermott K, Shaw C, Demchak J: Validity of functional stability limits as a measure of balance in adults aged 23-73 years. Ergonomics 2007, 50:631-646.

17. Gauthier C, Gagnon D, Grangeon M, Jacquemin G, Nadeau S, Masani K, Popovic MR: Comparison of multidirectional seated postural stability between individuals with spinal cord injury and able-bodied individuals. J Rehabil Med 2013, 45:47-54.

18. Gauthier C, Gagnon D, Jacquemin G, Duclos C, Masani K, Popovic MR: Which trunk inclination directions best predict multidirectional-seated limits of stability among individuals with spinal cord injury? I Spinal Cord Med 2012, 35:343-350.

19. Vette $A H$, Masani $K$, Sin V, Popovic MR: Posturographic measures in healthy young adults during quiet sitting in comparison with quiet standing. Med Eng Phys 2010, 32:32-38.

20. Kirshblum SC, Burns SP, Biering-Sorensen F, Donovan W, Graves DE, Jha A, Johansen M, Jones L, Krassioukov A, Mulcahey MJ, et al: International standards for neurological classification of spinal cord injury (revised 2011). J Spinal Cord Med 2011, 34:535-546.

21. Lam T, Noonan VK, Eng JJ, Team SR: A systematic review of functional ambulation outcome measures in spinal cord injury. Spinal Cord 2008, 46:246-254.

22. Forth $\mathrm{KE}$, Fiedler MJ, Paloski WH: Estimating functional stability boundaries for bipedal stance. Gait Posture 2011, 33:715-717.

23. Cohen J: Statistical power analysis for the behavioral sciences. 2nd edition. Hillsdale, NJ: L. Erlbaum Associates; 1988

24. Portney LG, Watkins MP: Foundations of clinical research : applications to practice. 3rd edition. Upper Saddle River, N.J: Pearson/Prentice Hall; 2009.

25. Ondo W, Warrior D, Overby A, Calmes J, Hendersen N, Olson S, Jankovic J: Computerized posturography analysis of progressive supranuclear palsy: a case-control comparison with Parkinson's disease and healthy controls. Arch Neurol 2000, 57:1464-1469.

26. Wang TY, Lin Sl: Sensitivity of plantar cutaneous sensation and postural stability. Clin Biomech 2008, 23:493-499.

27. Meyer PF, Oddsson LI, De Luca CJ: The role of plantar cutaneous sensation in unperturbed stance. Exp Brain Res 2004, 156:505-512.

28. Horlings $C G$, Kung UM, van Engelen BG, Voermans NC, Hengstman GJ, van der Kooi AJ, Bloem BR, Allum JH: Balance control in patients with distal versus proximal muscle weakness. Neuroscience 2009, 164:1876-1886.

29. Horlings CG, van Engelen BG, Allum JH, Bloem BR: A weak balance: the contribution of muscle weakness to postural instability and falls. Nat Clin Pract Neurol 2008, 4:504-515.

30. Marigold DS, Eng JJ, Tokuno CD, Donnelly CA: Contribution of muscle strength and integration of afferent input to postural instability in persons with stroke. Neurorehabil Neural Repair 2004, 18:222-229. 
31. Roerdink M, Hlavackova P, Vuillerme N: Effects of plantar-flexor muscle fatigue on the magnitude and regularity of center-of-pressure fluctuations. Exp Brain Res 2011, 212:471-476.

32. Forrest GF, Lorenz DJ, Hutchinson K, Vanhiel LR, Basso DM, Datta S, Sisto SA, Harkema SJ: Ambulation and balance outcomes measure different aspects of recovery in individuals with chronic, incomplete spinal cord injury. Arch Phys Med Rehabil 2012, 93:1553-1564.

33. Torres-Oviedo $G$, Ting LH: Muscle synergies characterizing human postural responses. J Neurophysiol 2007, 98:2144-2156.

34. Liston RA, Brouwer BJ: Reliability and validity of measures obtained from stroke patients using the Balance Master. Arch Phys Med Rehabil 1996, 77:425-430.

35. Robinett CS, Vondran MA: Functional ambulation velocity and distance requirements in rural and urban communities. A clinical report. Phys Ther 1988, 68:1371-1373.

36. Sayenko DG, Alekhina MI, Masani K, Vette AH, Obata H, Popovic MR, Nakazawa K: Positive effect of balance training with visual feedback on standing balance abilities in people with incomplete spinal cord injury. Spinal Cord 2010, 48:886-893.

doi:10.1186/1743-0003-11-8

Cite this article as: Lemay et al: Center-of-pressure total trajectory length is a complementary measure to maximum excursion to better differentiate multidirectional standing limits of stability between individuals with incomplete spinal cord injury and able-bodied individuals. Journal of NeuroEngineering and Rehabilitation 2014 11:8.

\section{Submit your next manuscript to BioMed Central and take full advantage of:}

- Convenient online submission

- Thorough peer review

- No space constraints or color figure charges

- Immediate publication on acceptance

- Inclusion in PubMed, CAS, Scopus and Google Scholar

- Research which is freely available for redistribution 